\title{
The Molecular Probes Handbook. A Guide to Fluorescent Probes and Labeling Technologies
}

\author{
(I. Johnson and M. Spence (eds.) 11th Edition, Life Technologies, 2010, 1060 p., \$100)
}

DOI: $10.1134 / \mathrm{S} 0006297911110101$

This book is a comprehensive resource for fluorescence technology that is continually updated. It contains detailed information about the thousands of Molecular Probes products available for fluorescence and imaging applications. The book is divided into six main parts, with a total of 23 chapters, each discussing a product group with common properties or applications. Each chapter is further subdivided into sections, grouping products by more narrowly defined properties or applications. If a product has multiple applications, it may appear in more than one location.

Part I highlights properties of fluorescent labels including their properties and labeling chemistries. This part contains a section with an introduction to fluorescence techniques and five chapters (1-5) devoted to fluorophores and their amine-reactive derivatives, thiol-reactive probes, chemistry and functional group modifications, biotin and hapten derivatives, and crosslinking and photoactivatable reagents.

Part II is devoted to detection of biomolecules: signal amplification and secondary detection. This part consists of two chapters ( 6 and 7 ), which describe ultrasensitive detection technology and antibodies, avidins and lectins.

Part III (chapters 8-10) describes technique of detecting cell components: nucleic acid and protein analysis. The final chapter of this part highlights enzyme substrates and assays.
Part IV collects four chapters (11-14) with the following topics: probes for cytoskeletal proteins, probes for organelles, probes for lipids and membranes, and fluorescent tracers of cell morphology and fluid flow.

Part V describes fluoro-probes as tools for study of cell function: from cell viability to ion flux. This part consists of eight chapters (15-22) with the following topics: assays for cell viability, proliferation, and function, probes for endocytosis, receptors and ion channels, probes for signal transduction, reactive oxygen species, indicators for $\mathrm{Ca}^{2+}, \mathrm{Mg}^{2+}, \mathrm{Zn}^{2+}$ and other metal ions, $\mathrm{pH}$ indicators, indicators for $\mathrm{Na}^{+}, \mathrm{K}^{+}, \mathrm{Cl}^{-}$and miscellaneous ions, and probes for membrane potential.

Part VI (chapter 23) highlights integrating technology and instrumentation and focuses on antifades and other tools for fluorescence.

At the end of the book there is an appendix with trademark information, limited use label licenses, master product and catalog number lists, and subject index.

The book is prepared by various experts including chemists, cell and molecular biologists, and electrical, mechanical, optical, and software engineers from all over the world. It is very comprehensive handbook for many specialists working in biochemistry, molecular biology, biotechnology, molecular medicine, and other branches of research.

An online version of the handbook is also available at Molecular Probes website (www.invitrogen.com/probes).

Doctor of Biological Sciences G. Ya. Wiederschain 